\title{
Preserving universal resources for one-way quantum computing
}

\author{
Tetsufumi Tanamoto, ${ }^{1}$ Daniel Becker, ${ }^{2}$ Vladimir M. Stojanović, ${ }^{2}$ and Christoph Bruder ${ }^{2}$ \\ ${ }^{1}$ Corporate $R \& D$ center, Toshiba Corporation, Saiwai-ku, Kawasaki 212-8582, Japan \\ ${ }^{2}$ Department of Physics, University of Basel, Klingelbergstrasse 82, CH-4056 Basel, Switzerland
}

(Dated: August 15, 2018)

\begin{abstract}
The common spin Hamiltonians such as the Ising, $X Y$, or Heisenberg model do not have eigenstates that are suitable resources for measurement-based quantum computation. Various highlyentangled many-body states have been suggested as a universal resource for this type of computation, however, it is not easy to preserve these states in solid-state systems due to their short coherence times. To solve this problem, we propose a scheme for generating a Hamiltonian that has a cluster state as ground state. Our approach employs a series of pulse sequences inspired by established NMR techniques and holds promise for applications in many areas of quantum information processing.

PACS numbers: 03.67.Lx,03.67.Pp,03.67.Ac
\end{abstract}

\section{INTRODUCTION}

Measurement-based quantum computation (MQC) is a new computing paradigm [1]. Of particular interest are universal resources of one-way quantum computation, a MQC scheme that requires only local measurements [2]. In the original scheme of one-way quantum computing, one initially creates a many-qubit cluster state by applying phase gates or equivalent gate operations which can be realized using the Ising interaction between qubits. Many promising methods to generate cluster states using solid-state qubits have been proposed 3 [ [5]. However, since these states are not the ground states of spin Hamiltonians with typical qubit-qubit interactions of Ising, $X Y$, and Heisenberg form [6], preserving them against the time evolution generated by these spin Hamiltonians remains a critical issue.

One of the established universal resources are twodimensional (2D) cluster states. Another promising candidate is the Affleck-Kennedy-Lieb-Tasaki (AKLT) state on the honeycomb lattice [7], a resonance valence bond (RVB) type state which is a special projected entangled pair state (PEPS) 8, 9]. Yet, the AKLT state requires non-trivial Hamiltonians with spin greater than $1 / 2$, which are not easy to realize in solid-state systems.

In this paper, we present a new method for preserving initially prepared cluster states. Our approach relies on manipulating a two-body Hamiltonian using pulsesequence techniques developed in the nuclear magnetic resonance (NMR) context [10, 11]. We show that, starting from the Ising and $X Y$ models, one can induce an effective dynamics described by a stabilizer Hamiltonian [2]

$$
H_{\mathrm{stab}}=-\sum_{i} K_{i}
$$

where $K_{i}=\sigma_{i}^{x} \bigotimes_{j \in \operatorname{nbhd}(i)} \sigma_{j}^{z}$ are the correlation operators and the direct product runs over all nearest neighbors of the lattice site $i$ ( $\sigma_{i}^{x}$ and $\sigma_{j}^{z}$ are the Pauli matrices). Combined with cluster state generation methods [1, 4], our scheme facilitates stable one-way quantum computing.

We assume the original Hamiltonian to be of the form $H=H_{0}+H_{\text {int }}$ where

$$
H_{0}=\sum_{i}\left(\Omega_{i} \sigma_{i}^{x}+\varepsilon_{i} \sigma_{i}^{z}\right)
$$

is a single-qubit part and $H_{\text {int }}$ the interaction part. We take $H_{\text {int }}$ to be of Ising $H_{\text {Ising }}=\sum_{i<j} J_{i j} \sigma_{i}^{z} \sigma_{j}^{z}, X Y$ $H_{\mathrm{XY}}=\sum_{i<j} J_{i j}[X Y]_{i j}$, and Heisenberg form $H_{\mathrm{H}}=$ $\sum_{i<j} J_{i j}[X Y Z]_{i j}$. In this paper, $J_{i j}=J$ if $i$ and $j$ are nearest neighbors and $J_{i j}=0$ otherwise. We use the shorthands $[X Y]_{i j} \equiv \sigma_{i}^{x} \sigma_{j}^{x}+\sigma_{i}^{y} \sigma_{j}^{y}$ and $[X Y Z]_{i j} \equiv$ $\sigma_{i}^{x} \sigma_{j}^{x}+\sigma_{i}^{y} \sigma_{j}^{y}+\sigma_{i}^{z} \sigma_{j}^{z}$, and set $\hbar=1$.

Note that a single correlation operator can be obtained using a single-qubit Hamiltonian. For example in a onedimensional (1D) qubit array, $K_{2}=\sigma_{2}^{x} \sigma_{1}^{z} \sigma_{3}^{z}$ can be generated by the time evolution operator $e^{i(\pi / 2)\left(\sigma_{1}^{z}+\sigma_{2}^{x}+\sigma_{3}^{z}\right)}$. However, it is not evident how to obtain a sum like $K_{2}+K_{3}$ from the single-qubit Hamiltonian.

Most fabricated solid-state qubit systems are nanodevices, because a smaller size makes them more robust to decoherence. An example are quantum dot systems where smaller dots have larger energy-level spacing. Since with diminishing size it becomes difficult to address these devices individually, it is of interest to consider switching on/off $H_{0}$ and $H_{\text {int }}$ independently. We will show that, by using appropriate pulse sequences, this is possible even if we start from an always-on Hamiltonian [12].

\section{METHOD FOR PRESERVING DESIRABLE STATES}

Desirable quantum states are preserved by a pulse sequence that is familiar in the NMR context [10]. We assume that each pulse is sufficiently strong such that interactions between qubits can be neglected during the pulse sequences. The time evolution of the system is described by the density operator $\rho(t)$ whose time dependence is given by $\rho(t)=\exp (-i H t) \rho(0) \exp (i H t)$ for 
time-independent $H$. It is convenient to use the following schematic notation for this evolution: $\rho(0) \stackrel{t H}{\longrightarrow} \rho(t)$. Then the process

$$
\rho(0) \stackrel{\tau_{1} H_{\text {int }}}{\longrightarrow} \stackrel{\tau H_{s}}{\longrightarrow} \stackrel{-\tau_{1} H_{\text {int }}}{\longrightarrow} \rho(t)
$$

for $\tau_{1}=\pi /(4 J)$ corresponds to $\rho(0) \stackrel{\tau H_{\text {stab }}}{\longrightarrow} \rho(t)$ where $t=\tau+\pi /(2 J)$ and $\tau$ can be chosen arbitrarily. Note that at the physical time $t$ the state of the system is obtained from the initial one by the time-evolution operator

$$
e^{-i \tau H_{\mathrm{stab}}}=\left.e^{-i \theta \sum_{i} \sigma_{i}^{z} \sigma_{i+1}^{z}} e^{-i \tau H_{\mathrm{s}}} e^{i \theta \sum_{i} \sigma_{i}^{z} \sigma_{i+1}^{z}}\right|_{\theta=\pi / 4} .
$$

Thus, as illustrated by Fig. 1(a), $H_{\text {stab }}$ becomes the effective system Hamiltonian. Its ground state is the originally prepared cluster state, which is therefore preserved.

\section{A. Ising model}

We now show how to construct the stabilizer Hamiltonian using the relation

$$
\begin{aligned}
& e^{-i \theta \sigma_{1}^{z} \sigma_{2}^{z}} \sigma_{1}^{x} e^{i \theta \sigma_{1}^{z} \sigma_{2}^{z}}=\cos (2 \theta) \sigma_{1}^{x}+\sin (2 \theta) \sigma_{1}^{y} \sigma_{2}^{z}, \\
& e^{-i \theta \sigma_{1}^{z} \sigma_{2}^{z}} \sigma_{1}^{y} e^{i \theta \sigma_{1}^{z} \sigma_{2}^{z}}=\cos (2 \theta) \sigma_{1}^{y}-\sin (2 \theta) \sigma_{1}^{x} \sigma_{2}^{z} .
\end{aligned}
$$

An important consequence of these equations is that, for $\theta=\pi / 4$, we can increase the order of the Paulimatrix terms as in $e^{-i \frac{\pi}{4} \sigma_{1}^{z} \sigma_{2}^{z}} \sigma_{1}^{x} e^{i \frac{\pi}{4} \sigma_{1}^{z} \sigma_{2}^{z}}=\sigma_{1}^{y} \sigma_{2}^{z}$ and $e^{-i \frac{\pi}{4} \sigma_{1}^{z} \sigma_{2}^{z}} \sigma_{1}^{y} e^{i \frac{\pi}{4} \sigma_{1}^{z} \sigma_{2}^{z}}=-\sigma_{1}^{x} \sigma_{2}^{z}$. For a 1D $N$-qubit chain, the starting single-qubit Hamiltonian is given by

$$
H_{\mathrm{s}}=\Omega\left(\sigma_{1}^{y}+\sum_{i=2}^{N-1} \sigma_{i}^{x}+\sigma_{N}^{y}\right) .
$$

By switching the interaction, the 1D stabilizer Hamiltonian is realized according to

$$
H_{\mathrm{stab}}=\left.e^{-i \theta \sum_{i} \sigma_{i}^{z} \sigma_{i+1}^{z}} H_{\mathrm{s}} e^{i \theta \sum_{i} \sigma_{i}^{z} \sigma_{i+1}^{z}}\right|_{\theta=\pi / 4},
$$

the equivalent of Eq. (44) at the Hamiltonian level. As an example, for $N=3$ qubits, starting from $H_{\mathrm{s}}=\Omega\left(\sigma_{1}^{y}+\right.$ $\left.\sigma_{2}^{x}+\sigma_{3}^{y}\right)$ we obtain $H_{\text {stab }}=\Omega\left(-\sigma_{1}^{x} \sigma_{2}^{z}-\sigma_{1}^{z} \sigma_{2}^{x} \sigma_{3}^{z}-\sigma_{3}^{x} \sigma_{2}^{z}\right)$. If the system of $N$ qubits has periodic boundary conditions, we start from the Hamiltonian $H_{\mathrm{S}}=\Omega \sum_{i=1}^{N} \sigma_{i}^{x}$. Since Ising-type interaction terms commute and the timeevolution operator $\exp \left(i(\pi / 4) \sum_{i} \sigma_{i}^{z} \sigma_{i+1}^{z}\right)$ in (17) factorizes, this process can be straightforwardly extended to $2 \mathrm{D}$ and $3 \mathrm{D}$ qubit systems, thus realizing the universal resource discussed in the introduction. Consequently, for Ising interactions, we can construct the stabilizer Hamiltonian by switching on $H_{\text {Ising }}$ only once.

\section{B. $X Y$ model}

Next, we show how to generate the stabilizer Hamiltonian using the $X Y$ interaction, assuming that $H_{0}$ and $H_{\text {int }}$ can be switched on/off independently.

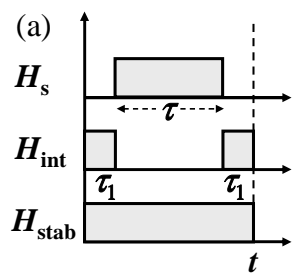

(b)

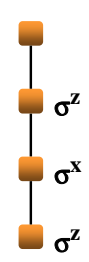

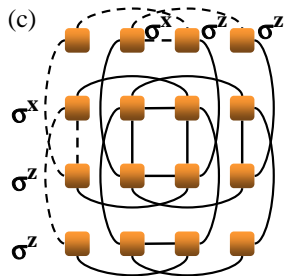

FIG. 1: (a) Switching on/off the parts $H_{s}$ and $H_{\text {int }}$ of the Hamiltonian $H=H_{s}+H_{\text {int }}$ gives rise to an effective time evolution described by the stabilizer Hamiltonian $H_{\text {stab }}$ [see Eq. (4)] whose ground state is a cluster state. (b) Graph representation of a one-dimensional 4-qubit (squares) cluster state stabilized by the Ising interaction. (c) Two-dimensional $4 \times 4$ twisted cluster state stabilized by the $X Y$ interaction, a universal resource for one-way quantum computation. The dashed lines and Pauli operators in each direction illustrate the twistedness of the state and the corresponding stabilizer Hamiltonian.

The stabilizer Hamiltonian is formed step by step by bonding the nearest-neighbor operators. This is because the $X Y$ interactions do not commute, $\left[[X Y]_{i-1, i},[X Y]_{i, i+1}\right] \neq 0$. We start from

$$
\begin{aligned}
e^{-i \theta[X Y]_{12}} \sigma_{1}^{x} e^{i \theta[X Y]_{12}} & =\cos (2 \theta) \sigma_{1}^{x}-\sin (2 \theta) \sigma_{1}^{z} \sigma_{2}^{y} \\
e^{-i \theta[X Y]_{12}} \sigma_{1}^{y} e^{i \theta[X Y]_{12}} & =\cos (2 \theta) \sigma_{1}^{y}+\sin (2 \theta) \sigma_{1}^{z} \sigma_{2}^{x} \\
e^{-i \theta[X Y]_{12}} \sigma_{1}^{z} e^{i \theta[X Y]_{12}} & =\cos ^{2}(2 \theta) \sigma_{1}^{z}+\sin ^{2}(2 \theta) \sigma_{2}^{z} \\
& +\frac{1}{2} \sin (4 \theta)\left[\sigma_{1}^{x} \sigma_{2}^{y}-\sigma_{1}^{y} \sigma_{2}^{x}\right]
\end{aligned}
$$

For $\theta=\pi / 4$, these transformations increase the order of the Pauli-matrix terms as $\sigma_{1}^{x} \rightarrow-\sigma_{1}^{z} \sigma_{2}^{y}$ and $\sigma_{1}^{y} \rightarrow \sigma_{1}^{z} \sigma_{2}^{x}$. For $\sigma_{1}^{z}$ one obtains $\sigma_{1}^{z} \rightarrow \sigma_{2}^{z}$.

We now show how to construct a $2 \mathrm{D}$ stabilizer Hamiltonian. First we construct the 1D stabilizer Hamiltonian, starting from

$$
H_{\mathrm{s}}=\Omega\left(-\sigma_{1}^{x}+\sigma_{2}^{y}-\sum_{i=3, N-2} \sigma_{i}^{x}+\sigma_{N-1}^{y}-\sigma_{N}^{x}\right) .
$$

In the specific case of six qubits in 1D, by applying Eq. (8) to $[X Y]_{12},[X Y]_{34}$, and $[X Y]_{56}$, we obtain:

$$
\begin{aligned}
e^{-i S_{1}} H_{\mathrm{s}} e^{i S_{1}} & =\Omega\left(\sigma_{1}^{z} \sigma_{2}^{y}+\sigma_{2}^{z} \sigma_{1}^{x}+\sigma_{3}^{z} \sigma_{4}^{y}+\sigma_{4}^{z} \sigma_{3}^{y}\right. \\
& \left.+\sigma_{5}^{z} \sigma_{6}^{y}+\sigma_{6}^{z} \sigma_{5}^{y}\right)
\end{aligned}
$$

where $S_{1}=\frac{\pi}{4} \sum_{l=1,3}[X Y]_{\{2 l-1,2 l\}}$. Repeating this step with $S_{2}=\frac{\pi}{4} \sum_{l=1,2}[X Y]_{\{2 l, 2 l+1\}}$, we get the $1 \mathrm{D}$ stabilizer Hamiltonian $H_{1 \mathrm{D}}=e^{-i S_{2}} e^{-i S_{1}} H_{\mathrm{S}} e^{i S_{1}} e^{i S_{2}}$ that reads explicitly

$$
\begin{aligned}
H_{1 \mathrm{D}} & =\Omega\left(\sigma_{1}^{z} \sigma_{2}^{z} \sigma_{3}^{x}+\sigma_{3}^{z} \sigma_{1}^{x}+\sigma_{2}^{z} \sigma_{4}^{z} \sigma_{5}^{x}+\sigma_{5}^{z} \sigma_{3}^{z} \sigma_{2}^{x}\right. \\
& \left.+\sigma_{4}^{z} \sigma_{6}^{x}+\sigma_{6}^{z} \sigma_{5}^{z} \sigma_{4}^{x}\right) .
\end{aligned}
$$

This Hamiltonian is twisted in the sense of [4], i.e., the site indices of the corresponding cluster state are obtained by the permutation $(2,3)(4,5) \ldots(N-2, N-1)$ 
(cyclic notation), for a chain of $N$ qubits where $N$ is even, see Fig. 1(b,c).

The next step in the construction of the 2D stabilizer Hamiltonian is to construct a ladder Hamiltonian by bonding nearest-neighbor sites on adjacent chains $a$ and $b$, in which all the bondings between qubits $i a$ and $i b$ are carried out simultaneously:

$$
\begin{aligned}
H_{\text {ladder }} & =\Omega\left(-\sigma_{1 a}^{z} \sigma_{2 a}^{z} \sigma_{b 3}^{z} \sigma_{3 a}^{y}-\sigma_{3 a}^{z} \sigma_{1 b}^{z} \sigma_{1 a}^{y}\right. \\
& -\sigma_{2 a}^{z} \sigma_{4 a}^{z} \sigma_{55}^{z} \sigma_{5 a}^{y}-\sigma_{5 a}^{z} \sigma_{3 a}^{z} \sigma_{2 b}^{z} \sigma_{2 a}^{y}-\sigma_{4 a}^{z} \sigma_{6 a}^{y} \sigma_{6 b}^{z} \\
& \left.-\sigma_{6 a}^{z} \sigma_{5 a}^{z} \sigma_{4 b}^{z} \sigma_{4 a}^{y}\right)+(a \leftrightarrow b) .
\end{aligned}
$$

A 2D stabilizer Hamiltonian is produced by connecting the above two ladder Hamiltonians with the interaction between the two ladders. For example, when we prepare two ladders of length 4 such as in Fig. 1(c) and connect them vertically, we obtain a $4 \times 4$ stabilizer Hamiltonian.

\section{Heisenberg model}

For the Heisenberg interaction, we can construct only a two-qubit stabilizer Hamiltonian (note that the same is true for the $X X Z$ interaction). The basic relation is

$$
\begin{aligned}
& e^{-i \theta[X Y Z]_{12}} \sigma_{1}^{y} e^{i \theta[X Y Z]_{12}}=\cos ^{2}(2 \theta) \sigma_{1}^{y} \\
& +\sin ^{2}(2 \theta) \sigma_{2}^{y}+\frac{1}{2} \sin (4 \theta)\left(\sigma_{1}^{x} \sigma_{2}^{y}-\sigma_{1}^{y} \sigma_{2}^{x}\right) .
\end{aligned}
$$

For the Ising and $X Y$ models, we can eliminate the single Pauli matrix terms leaving the interaction terms [see Eqs. (5) and (8)]. However, in Eq. (13), if we set $\sin (2 \theta)=0$ or $\cos (2 \theta)=0$, we also eliminate the $\sigma_{1}^{x} \sigma_{2}^{y}-\sigma_{1}^{y} \sigma_{2}^{x}$ term. This is because the Heisenberg interaction contains terms in all three spatial directions [4]. In the case of two qubits, we obtain $H=\Omega\left(\sigma_{1}^{z} \sigma_{2}^{x}-\sigma_{1}^{x} \sigma_{2}^{z}\right)$ from the initial Hamiltonian $H_{s}=\Omega\left(\sigma_{1}^{y}-\sigma_{2}^{y}\right)$ by using Eq. (13) for $\theta=\pi / 8$. By applying a $\pi$-rotation, we obtain the two-qubit stabilizer Hamiltonian $H=$ $\Omega\left(\sigma_{1}^{z} \sigma_{2}^{x}+\sigma_{1}^{x} \sigma_{2}^{z}\right)$.

\section{MANIPULATION OF ALWAYS-ON HAMILTONIAN}

The scheme discussed up to now relies on switching on/off the single-qubit Hamiltonian $H_{0}$ [see Eq. (2)] and the Ising or $X Y$ interaction part $H_{\text {int }}$ separately. There is a number of schemes for switching on/off interactions between qubits (see, e.g., 13 15]). However, they make the system more complicated and require additional overhead.

Here, we solve this problem by demonstrating how to extract $H_{0}$ and $H_{\text {int }}$ by using appropriate pulse sequences. We illustrate the idea using the standard NMR Hamiltonian $H_{\mathrm{nmr}}=\sum_{i} \varepsilon_{i} \sigma_{i}^{z}+\sum_{i} J \sigma_{i}^{z} \sigma_{i+1}^{z}$ which has the property that $\left[H_{0}, H_{\text {int }}\right]=0$. In this case, $H_{0}$ and $H_{\text {int }}$ can be switched on/off by using a simple pulse sequence. The interaction part $H_{\text {Ising }}$ can be extracted by using two sandwiched $\pi$-pulses such as $\exp \left(i \tau H_{\text {Ising }}\right)=$ $e^{-i(\pi / 2) \sum_{j} \sigma_{j}^{y}} e^{i(\tau / 2) H_{\mathrm{nmr}}} e^{i(\pi / 2) \sum_{j} \sigma_{j}^{y}} e^{i(\tau / 2) H_{\mathrm{nmr}}}$. On the other hand, two steps are required to obtain $H_{0}$. Let us consider a 1D qubit chain. By applying a $\pi$-pulse about the $x$-axis (denoted by $(\pi)_{x}$ ) to all the qubits on the even sites, we obtain $e^{-i(\pi / 2) \sum_{i} \sigma_{2 i}^{y}} e^{i(\tau / 2) H_{\mathrm{nmr}}} e^{i(\pi / 2) \sum_{i} \sigma_{2 i}^{y}} e^{i(\tau / 2) H_{\mathrm{nmr}}}=$ $e^{i \tau \sum_{i} \Omega \sigma_{2 i-1}^{z}}$. Similarly, we obtain $e^{i \tau \sum_{i} \Omega \sigma_{2 i-1}^{z}}$ by applying a $(\pi)_{x}$-pulse to all the qubits on the odd sites. Combining these two processes yields $H_{0}$. This method is easily generalized to $2 \mathrm{D}$ or $3 \mathrm{D}$ qubit arrays.

If $\left[H_{0}, H_{\text {int }}\right] \neq 0$, this NMR method cannot be used. Even in this case, $H_{0}$ and $H_{\text {int }}$ can be extracted separately. The idea follows from average Hamiltonian theory which is based on the Baker-Campbell-Hausdorff $(\mathrm{BCH})$ formula for the expansion of $e^{A} e^{B}[10]$. A stroboscopic application of the Hamiltonian designed by a series of short pulses can reduce or eliminate unwanted terms, if $\Omega \tau, J \tau \ll 1$. First we extract $H_{\text {int }}$ by setting $A=h_{0}+h_{1}$ and $B=-h_{0}+h_{1}$ in the $\mathrm{BCH}$ formula, where $h_{0} \equiv H_{0} \tau$ and $h_{1} \equiv H_{\text {int }} \tau . \quad B$ is realized by applying a $(\pi)_{y}$-pulse on every qubit. From the BCH formula, we obtain

$$
e^{A} e^{B} \approx \exp \left(2 h_{1}+\left[h_{0}, h_{1}\right]+\frac{1}{3}\left[h_{0},\left[h_{0}, h_{1}\right]\right]\right) .
$$

The exponent corresponds to a third-order expansion in $\Omega \tau$ for $\Omega \tau \ll 1$. If we repeat this operation $n$ times like $e^{A} e^{B} e^{A} e^{B} \cdots e^{A} e^{B}=\left(e^{A} e^{B}\right)^{n}$ such that $n \Omega \tau=\pi / 4$, the $k$-th term is of order $[\pi /(4 n)]^{k}$. Therefore, $H_{0}$ is canceled, and we obtain only $H_{\text {int }}$ in this order. When we apply

$$
e^{A} e^{B} e^{B} e^{A} \approx \exp \left(4 h_{1}-\frac{5}{3}\left[h_{1},\left[h_{0}, h_{1}\right]\right]+\frac{1}{3}\left[h_{0},\left[h_{0}, h_{1}\right]\right]\right),
$$

we can eliminate the second term in Eq. (14). In the limit $n \rightarrow \infty$ under the condition of $n \Omega \tau=\pi / 4, H_{0}$ is exactly eliminated. The extraction of $H_{0}$ can be achieved analogously. Moreover, as shown in [16], if the $k$ th-order term is the first nonvanishing correction, the decay rate $T_{2}$ of the qubit system is enlarged according to $T_{2}^{\prime} \propto$ $T_{2}(k+1) !\left(T_{2} / t_{c}\right)^{k}$ as long as $T_{2}>t_{c}$ where $t_{c}$ is the time required for each single step [ $A$ and $B$ in Eq. (14)].

For the $X Y$ model, we have to switch off subsets of $H_{\text {int }}$ corresponding to $S_{1}$ and $S_{2}$, as discussed after Eq. (10). This is equivalent to choosing $A$ and $B$ appropriately: e.g., for the $1 \mathrm{D}$ chain, $A=H_{\mathrm{int}}=h_{1 e}+h_{1 o}$ and $B=$ $h_{1 e}-h_{1 o}$ where $h_{1 e}=J \tau\left([X Y]_{23}+[X Y]_{45}+[X Y]_{67}+..\right)$ and $h_{1 o}=J \tau\left([X Y]_{12}+[X Y]_{34}+[X Y]_{56}+..\right)$. That means, $B$ is generated by applying $\pi$ pulses to qubits $2,3,6,7,10,11, \ldots$.

Let us consider the 1D $X Y$ model with $\varepsilon_{i}=0$ in Eq. (2). The following operation can be used to obtain $H_{0}$. (i) Applying a $(\pi)_{x}$-pulse to all the qubits on the even sites changes the sign of $\sum_{i} \sigma_{i}^{y} \sigma_{i+1}^{y}$. (ii) By further applying a $(\pi)_{y}$-pulse to the same subset of qubits, we obtain $B / \tau=\sum_{i} \Omega\left(\sigma_{2 i-1}^{x}-\sigma_{2 i}^{x}\right)-\sum_{i} J[X Y]_{i, i+1}$. As a result, we obtain $\Omega \sum_{i} \sigma_{2 i-1}^{x}$. Repeating the same operations with the qubits on the odd sites, we obtain 
$\Omega \sum_{i} \sigma_{2 i}^{x}$. For the Ising model, the process (i) is not required. For the Heisenberg model, the same procedure as in the case of the $X Y$ model does not eliminate the term $J \sum_{i} \sigma_{i}^{z} \sigma_{i+1}^{z}$. Thus, additional similar steps are required to eliminate this term.

\section{ROBUSTNESS}

Since a practical realization of these pulse sequences will not be free of imperfections, we now analyze the effect of pulse duration errors $\delta$. A central quantity will be the cluster-state fidelity $F_{\text {st }}(\tau)=\left|\left\langle\Psi_{00 \ldots 0}\left|U_{\tau}(\delta)\right| \Psi_{00 \ldots 0}\right\rangle\right|^{2}$ where $U_{\tau}(\delta)=$ $e^{-i \tau H_{\text {stab }}(\pi / 4+\delta / 4)}$. In the Ising case, $H_{\text {stab }}$ is given by Eq. (7), and an analogous equation in the $X Y$ case.

Let us consider the 1D Ising case. For $\theta=\pi / 4+\delta / 4$, the first-order correction to Eq. (7) reads

$$
H^{\prime}(\delta)=\frac{\delta}{2} \Omega\left[\sigma_{1}^{y}+\sigma_{N}^{y}+\sum_{1<i<N} \sigma_{i}^{y}\left(\sigma_{i-1}^{z}+\sigma_{i+1}^{z}\right)\right] .
$$

The effect of these terms is calculated from perturbation theory using the expressions for $\sigma_{i}^{\alpha}\left|\Psi_{00 . .0}\right\rangle$, where $\left|\Psi_{00 \ldots 0}\right\rangle$ is the initial cluster state, generated e.g. as proposed in Ref. [4], and $\alpha=x, y, z$ [17]. The lowest-order expression of the cluster-state fidelity reads $F_{\mathrm{st}}(\tau) \approx$ $\left[1+\Omega^{2} \tau^{2} \delta^{2}(N-1) / 2\right]^{-2}$, and the correction scales with $\delta^{2}$ which is a signature of the robustness of our method.

The simplest and most powerful method to further reduce the effect of pulse imperfections is the symmetrization of the pulse sequence frequently used in NMR 10 . We first note that Eq. (3) is equivalent to

$$
\rho(0) \stackrel{\tau_{1} H_{\text {int }}}{\longrightarrow} \stackrel{\frac{\tau}{2} H_{0}}{\longrightarrow}-\tau_{1} H_{\text {int }}-\stackrel{\tau_{1} H_{\text {int }}}{\longrightarrow} \stackrel{\tau}{\longrightarrow} H_{0} \stackrel{\tau_{1} H_{\text {int }}}{\longrightarrow} \rho(t),
$$

where, as before, $\tau_{1}=\pi /(4 J)$. The second half of this pulse sequence results in a perturbation term that has the opposite sign as compared to Eq. (16). Applying Eq. (14) leads to a cancellation of the firstorder perturbation term. If the original interval length $\tau$ is divided into an even number $n$ of subintervals, $\tau=n t_{c}$, the perturbation term is replaced by $\left[H_{\mathrm{stab}}, H^{\prime}\right] i \tau \delta /(4 n)$, and the fidelity $F_{\mathrm{sym}}^{n}$ is given by $F_{\text {sym }}^{n} \approx\left[1+\Omega^{4}\left(2 \tau^{2} / n\right)^{2} \delta^{2}(N-1) / 2\right]^{-2}$. Hence, the fidelity is improved, $F_{\mathrm{sym}}^{n}>F_{\mathrm{st}}$, if $n>2 \Omega \tau$.

These perturbative results are complemented by exact numerical calculations of $F_{\text {st }}(\tau)$ and the gate fidelity $F_{\mathrm{g}}(\tau)=2^{-N}\left|\operatorname{Tr} U_{\tau}^{\dagger}(0) U_{\tau}\left(\left\{\delta_{i}\right\}\right)\right|$ for $\tau=\pi /(4 \Omega)$ in systems with up to 10 qubits. Here, $\delta_{i}, i=1, \ldots, N-1$ corresponds to the qubit pair $(i, i+1)$. In both the Ising and $X Y$ case, we averaged these fidelities over 2000 random realizations of the $\delta_{i}$ taken from a Gaussian distribution with varying width $\sigma$. The results indicate that the method is rather robust even outside the regime where $\sigma$ is much smaller than $\pi / 4 \approx 0.78$. For instance, the $X Y$ model calculation shows that $F_{\text {st }}$ is bigger than $99 \%$ for

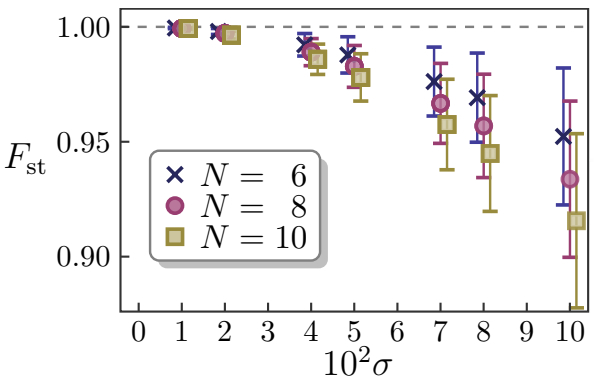

FIG. 2: Dependence of the cluster-state fidelity for $\tau=$ $\pi /(4 \Omega)$ on random errors in the pulse duration for onedimensional $X Y$ qubit arrays of length $N=6,8$, and 10 . The width of the Gaussian distribution is denoted by $\sigma$; the error bars indicate the standard deviation.

$\sigma \lesssim 0.04$, while $F_{g}$ can be bigger than $99 \%$ even for $\sigma$ as large as 0.07. The comparison between the two models shows that the robustness in the $X Y$ case (see Fig. 2) is somewhat better than in the Ising case.

\section{DISCUSSION AND CONCLUSION}

The distinguishing feature of the stabilizer Hamiltonians discussed here is that their ground states are directly related to the universal resource of measurementbased quantum computation. More generally, the stabilizer formalism has further applications in quantum information theory 18]. For instance, in quantum error-correcting codes [19], the stabilizer formalism is used to express codewords. The method illustrated in Eq. (3) can be used to obtain eigenvalues for the syndrome measurements in the process of detecting errors. Moreover, relations (5) and (8) can be used to effectively generate codewords in solid-state qubits. For example, the three-qubit GreenbergerHorne-Zeilinger (GHZ) state $(|000\rangle \pm|111\rangle) / \sqrt{2}$, which is used for the nine-qubit code, is effective generated by $e^{ \pm i(\pi / 4) \sigma_{1}^{x} \sigma_{2}^{y} \sigma_{3}^{x}}|000\rangle$, where $e^{i(\pi / 4) \sigma_{1}^{x} \sigma_{2}^{y} \sigma_{3}^{x}}=$ $e^{i(\pi / 2)\left(\sigma_{1}^{y}+\sigma_{3}^{y}\right)} e^{ \pm i(\pi / 4) \sigma_{1}^{z} \sigma_{2}^{y} \sigma_{3}^{z}} e^{-i(\pi / 2)\left(\sigma_{1}^{y}+\sigma_{3}^{y}\right)}$ is applied to the three-qubit array. The five-qubit code [19] can also be generated by using this method.

To conclude, we have shown that initially prepared cluster states, e.g., 2D cluster states that are universal resources of measurement-based quantum computation, can be preserved with high fidelity. This is achieved by inducing the effective dynamics of 2D stabilizer Hamiltonians by means of specially tailored pulse sequences, starting from natural qubit-qubit interactions. We have also shown how this procedure can be implemented in the case of always-on interactions. Our work will facilitate implementations of one-way quantum computing. 


\section{Acknowledgments}

We would like to thank J. Koga and F. Nori for discussions. This work was financially supported by the EU project SOLID, the Swiss SNF, the NCCR Nanoscience, and the NCCR Quantum Science and Technology.

(2010).

[12] R. Heule, C. Bruder, D. Burgarth, and V.M. Stojanović Phys. Rev. A 82, 052333 (2010); Eur. Phys. J. D 63, 41 (2011); V.M. Stojanović, A. Fedorov, A. Wallraff, and C. Bruder, Phys. Rev. B 85, 054504 (2012).

[13] D.V. Averin and C. Bruder, Phys. Rev. Lett. 91, 057003 (2003).

[14] A.O. Niskanen, Y. Nakamura, and J.S. Tsai, Phys. Rev. B 73, 094506 (2006).

[15] Y.X. Liu, L.F. Wei, J.S. Tsai, and F. Nori, Phys. Rev. Lett. 96, 067003 (2006); M. Grajcar, Y.X. Liu, F. Nori, and A.M. Zagoskin, Phys. Rev. B 74, 172505 (2006).

[16] U. Haeberlen and J.S. Waugh, Phys. Rev. 175, 453 (1968).

[17] H. Aschauer, W. Dür, and H.-J. Briegel, Phys. Rev. A 71, 012319 (2005).

[18] M.A. Nielsen and I.L. Chuang, Quantum Computation and Quantum Information (Cambridge University Press, Cambridge, 2000).

[19] D. Gottesman, Californian Institute of Technology, Pasadena, 1997, arXiv:quant-ph/9705052. 